\title{
Cannabis-Induced Brugada Syndrome Presenting as Cardiac Arrest
}

\author{
Tanya Doctorian* and Eric Chou \\ Department of Internal Medicine, Kaiser Permanente Fontana Medical Center, USA
}

Submission: May 09, 2017; Published: June 09, 2017

*Corresponding author: Tanya Doctorian, Department of Internal Medicine, Division of Cardiology, Kaiser Permanente Fontana Medical Center (909) 427-5000, 9961 Sierra Ave, Fontana, CA 92350, USA, Email: tanya.doctorian@kp.org

\begin{abstract}
Brugada syndrome $(\mathrm{BrS})$ is an inherited sodium channelopathy, which can precipitate malignant ventricular arrhythmias and sudden cardiac death. This is a 43-year-old male who presented with ventricular fibrillation cardiac arrest induced by cannabis in the setting of previously undiagnosed BrS. With the increasing prevalence of cannabis use, it is necessary to be aware of its potential adverse cardiovascular effects. This case highlights the diagnostic and clinical challenge of $\mathrm{BrS}$ and a raises the issue of cannabis as a potential provoking agent of malignant arrhythmias in BrS
\end{abstract}

Keywords: Brugada syndrome; Cannabis; Marijuana; Cardiac arrest

\section{Introduction}

Brugada syndrome (BrS) is an inherited sodium channelopathy, which can precipitate malignant ventricular arrhythmias and sudden cardiac death [1]. Characteristic electrocardiographic features without underlying structural heart disease make the diagnosis, and certain drugs can unmask the abnormality on electrocardiography (ECG). While sinus tachycardia is a known side effect of cannabis use, Brugada pattern on ECG and ventricular arrhythmias in the setting of BrS are much less known consequences and should be considered in young patients presenting with cardiac arrest without other risk factors. Despite increasing use of marijuana and evidence of its cardiovascular effects, there is a paucity of case reports and the pathogenesis of marijuana-induced BrS remains unknown. This case highlights the diagnostic and clinical challenge of BrS and a raises the issue of cannabis as a potential provoking agent of malignant arrhythmias in BrS.

\section{Case Presentation}

A 43-year-old male with no significant past medical history presented with ventricular fibrillation (VF) arrest two hours after smoking marijuana. The patient's fiancé witnessed his collapse from standing position to the floor and he was found unresponsive immediately thereafter with no pulse. She initiated compressions and an emergency medical service was called within seconds. He achieved return to spontaneous circulation after 4 shocks. He was intubated in the field for airway protection.

The patient did not have family history of cardiovascular disease or sudden cardiac death. He did not take any medications. He had occasional alcohol consumption, 17 pack-year smoking history, and weekly cannabis use. About 7 days leading to the cardiac arrest, the patient had been smoking marijuana several times per day, and consuming a high amount of caffeine and energy drinks due to increased stress at work. His fiancé reported that when he smoked cannabis, he would experience palpitations and lightheadedness.

In the emergency department, he was afebrile, hypotensive, and saturating $93 \%$ on $40 \%$ FiO2 on the ventilator. Cardiac and pulmonary exams were nonfocal. Workup revealed leukocytosis, elevated initial troponin and urine drug screen, which was positive for 9-carboxy tetrahydrocannabinol (THC) without presence of other drugs.

Initial ECG during admission revealed normal sinus rhythm with no ST-segment changes. Chest radiograph revealed patchy infiltrates in the right lower base, which resolved the next day. CT of the head revealed no evidence of acute intracranial 
hemorrhage, mass, or process. Transthoracic echocardiography revealed preserved left ventricular (LV) ejection fraction (60\%), normal LV cavity size, no wall motion abnormalities, no valvular disease, and no structural heart disease. He was started on clindamycin for presumed aspiration pneumonia and initiated on hypothermia protocol. The pulmonary opacities resolved the next day, suggesting pneumonitis rather than pneumonia. Respiratory and blood cultures were negative. Cardiac catheterization revealed no epicardial coronary artery disease. On hospital day 4, the patient was extubated. The patient's mental status improved over the next several days.

Cardiology attributed the patient's VF arrest to cannabis abuse and excessive stimulants. Electrophysiology was consulted for workup of implantable cardioverter-defibrillator (ICD) for secondary prevention of sudden cardiac death. During electrophysiology testing, the patient underwent procainamide challenge test, which unmasked Type I Brugada pattern on ECG. It was thought that his excess cannabis use in the setting of his underlying, previously undiagnosed BrS without other risk factors or triggers precipitated subsequent VF arrest. He underwent ICD placement, was started on quinidine, and referred to genetic testing. He was counseled on cannabis abstinence and the need for pyrolytics in setting of fevers. He was discharged home in stable condition.

Six months post-discharge, the patient was readmitted for VF storm evoking multiple ICD shocks during marijuana intoxication. He was afebrile at that time with no electrolyte abnormalities or evidence of infection. The ECG on admission depicted Type I Brugada pattern (Figure 1). Troponins were negative. With supportive therapy, the patient improved clinically and his ECG abnormality resolved. Given the temporal association with cannabis use and Type I Brugada pattern as well as the absence of other triggers such as fever or infection, he was advised to avoid further cannabis use.

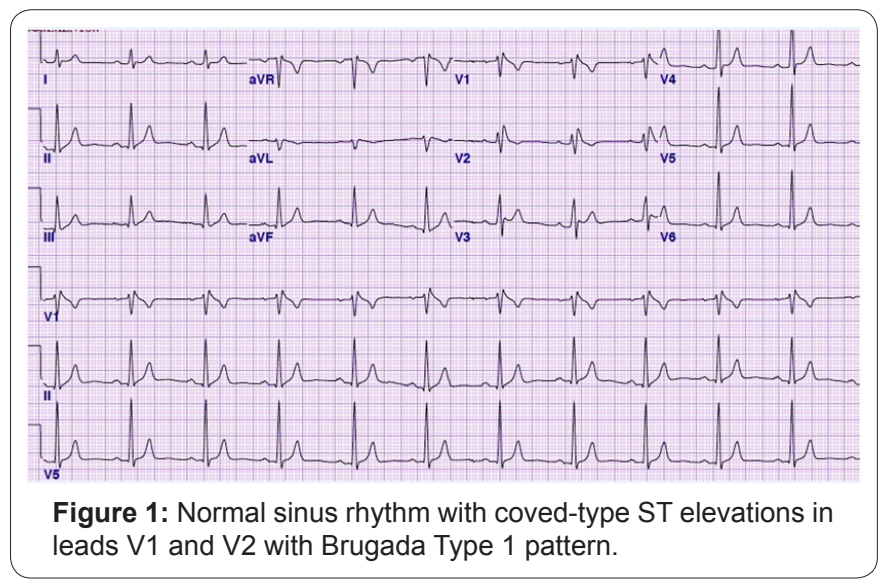

\section{Discussion}

Brugada syndrome is inherited in an autosomal dominant manner with a $50 \%$ risk of passing the mutation to each offspring, but there can also be variable penetrance in families [2]. About $1 \%$ of cases are de novo, or due to a new mutation in the affected individual. There are 19 known genetic mutations to date. The vast majority of patients with a genetic mutation have involvement of the SCN5A gene leading to accelerated sodium channel inactivation, precipitating characteristic Brugada ECG abnormalities, which can degenerate into malignant ventricular arrhythmias. BrS can manifest as syncope or cardiac arrest during the third or four decade of life, however the majority of patients are asymptomatic [3].

The diagnosis of Brugada syndrome is made with type I Brugada "coved-type" pattern (elevation of the J wave >or equal to $2 \mathrm{~mm}$ with a precordial lead) on ECG spontaneously, with administration of a sodium channel blocker (procainamide challenge testing), or with induction by a trigger such as fever or vagal stimulating medications [1]. The ECG changes can be accompanied by VF, ventricular tachycardia, family history of sudden cardiac death under 45 years of age, coved-type ECGs in relatives, syncope, or nocturnal agonal respiration [1]. BrS may manifest as type 2 pattern ("saddle-back" configuration) or type 3 pattern on ECG. About 25 to $30 \%$ of patients with Brugada pattern on ECG have an identifiable mutation with molecular testing. In drug-induced $\mathrm{BrS}$, it is unclear how much of a role genetic predisposition plays [4].

In patients with Brugada pattern on ECG, it is important to rule out other conditions that may produce similar electrocardiographic findings such as: acute myocardial infarction, pulmonary embolism, left ventricular hypertrophy, aortic dissection, hypothermia, electrolyte abnormalities (such as hypo- or hyperkalemia, or hypercalcemia), hemopericardium, or arrhythmogenic right ventricular cardiomyopathy $[1,4]$.

There are a few case reports describing symptomatic Brugada ECG pattern in the setting of cannabis intoxication, but cannabis is not included on the standard list of medications to avoid in BrS $[5,6]$. Given the temporal association between cannabis intoxication and type I pattern on ECG and later VF storm in our patient, it was thought that his excess cannabis use in the setting of his underlying, previously undiagnosed BrS without other risk factors or triggers precipitated subsequent VF arrest and VF storm. This case highlights the importance of recognizing cannabis use as a potential trigger of malignant arrhythmias in BrS in the absence of other obvious risk factors and structural heart disease. The mechanism by which cannabis may unmask Brugada pattern on ECG or induce VF in the setting of underlying BrS is largely unknown. However, it is proposed that the vagal stimulating effects of cannabis may precipitate Brugada pattern, as vagal stimulation is a known trigger of $\mathrm{BrS}$, and can degenerate into VF $[3,5,6]$.

While there is no known cure for BrS, the key strategies of Brugada management are prevention of malignant arrhythmias and patient education on triggers to avoid [1,3,7]. Triggers that should be avoided in BrS patients include antipsychotic medications (tricyclic antidepressants, lithium, fluoxetine), antihistamines, febrile illness, cocaine, sodium channel 
blockers, electrolyte abnormalities, and vagal stimulating agents [4]. We would also recommend caution with cannabis as a potential trigger of malignant arrhythmias in $\mathrm{BrS}$, as in our patient. According to the 2015 ESC Guidelines, cardiac arrest and sustained ventricular arrhythmias are Class I indications for ICD implantation in BrS patients [7]. ICD implantation is a Class IIa recommendation in patients with spontaneous Type 1 Brugada pattern on ECG and history of syncope, and a Class IIb recommendation in BrS patients with inducible VF $[3,6]$. Asymptomatic patients with type I Brugada pattern on ECG are at increased risk for a future adverse cardiovascular event, and ICD implantation should be considered on an individual basis. In BrS patients who have an ICD, quinidine, a class I antiarrhythmic, can be used to prevent recurrence of VF, as in our patient's case [7].

$\mathrm{BrS}$ is responsible for an estimated $20 \%$ of sudden cardiac death cases in patients with structurally normal hearts and about $72 \%$ of SCD events related to BrS occurred in asymptomatic people [3]. Thus, risk stratification in BrS patients is essential to prevent adverse cardiovascular outcomes. According to a recent meta-analysis, the incidence of malignant arrhythmias such as sustained ventricular tachycardia, appropriate ICD shocks or SCD was $13.5 \%$ per year in patients with a history of cardiac arrest, $3.2 \%$ in patients with syncope, and 1\% in asymptomatic patients [6]. A history of cardiac arrest, as in our patient, portends the highest risk of future malignant arrhythmias and SCD. Other predictors of future adverse events in BrS include a family history of SCD under 45 years of age, a reduced right ventricular EF, increased right ventricular pressures, Type I Brugada pattern on ECG, first degree atrioventricular block, and inducible sustained ventricular arrhythmias [3,6]. Clinicians must implement aggressive strategies to prevent adverse cardiovascular outcomes in vulnerable BrS patients.

\section{Conclusion}

While it is difficult to prove, this case raises suspicion of VF induced by cannabis in the setting of our patient's previously undiagnosed BrS without other risk factors. In patients presenting with cardiac arrest or syncope without known cardiovascular or structural heart disease, it is critical to consider workup for $\mathrm{BrS}$, as risk stratification, prevention counseling and ICD placement can be life-saving. Furthermore, this case raises suspicion of cannabis as a potential provoking agent of malignant arrhythmias in BrS and advises caution with cannabis in patients with BrS.

\section{References}

1. Antzelevitch C, Brugada P, Borggrefe M, Brugada J, Brugada R, et al. (2005) Brugada syndrome: report of the second consensus conference: endorsed by the Heart Rhythm Society and the European Heart Rhythm Association. Circulation 111(5): 659-670.

2. Juang JM, Horie M (2016) Genetics of Brugada syndrome. J Arrhythm $32(5): 418-425$.

3. Letsas KP, Georgopoulos S, Vlachos K, Karamichalakis N, Liatakis I, et al. (2016) Brugada Syndrome: Risk Stratification and Management. J Atr Fibrillation 9(2): 1413.

4. Yap YG, Behr ER, Camm AJ (2009) Drug-induced Brugada syndrome. Europace 11(8): 989-994.

5. Romero-Puche AJ, Trigueros-Ruiz N, Cerdan-Sanchez MC, PérezLorente F, Roldán D, et al. (2012) Brugada Electrocardiogram Pattern Induced by Cannabis. Rev Esp Cardiol 65(9): 851-861.

6. Daccarett M, Freih M, Machado C (2007) Acute cannabis intoxication mimicking brugada-like ST segment abnormalities. Int J Cardiol 199(2): 235-236.

7. Vohra J, Rajagopalan S (2015) Update on the Diagnosis and Management of Brugada Syndrome. Heart Lung Circ 24(12): 1141-1148.

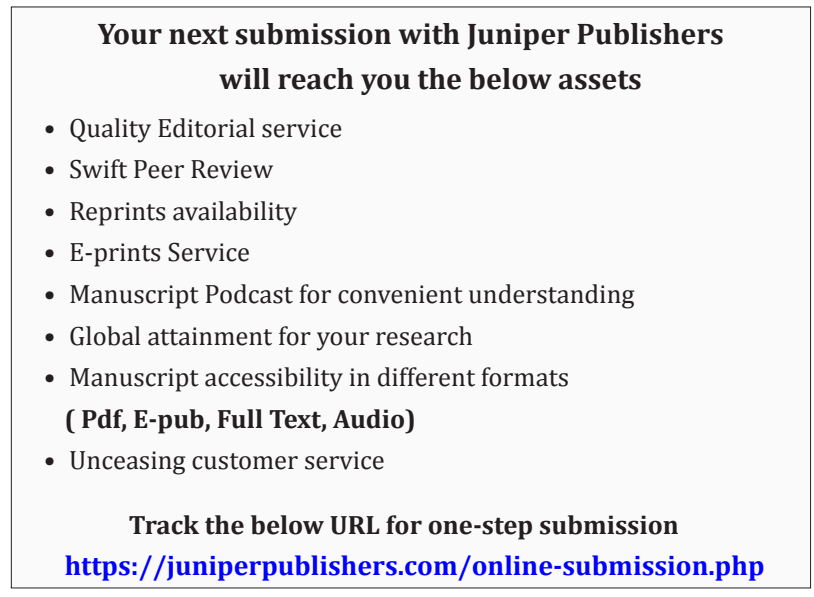

